\title{
Ultrasonic Detection using Wideband Discrete Wavelet Transform
}

\author{
F.K. Lam and C.K. Leung
}

\begin{abstract}
F.K. Lam: Department of Electrical and Electronic Engineering, The University of Hong Kong, Pokfulam Road, Hong Kong. email:fklam@eee.hku.hk

C.K. Leung: Center for Multimedia Signal Processing (CMSP) Department of Electronic and Information Engineering The Hong Kong Polytechnic University, Hung Hom, Hong Kong. email:enchikin@inet.polyu.edu.hk
\end{abstract}

\begin{abstract}
This paper describes the design of a wideband spatial processor for the detection of a straight-line object by an ultrasonic pulse-echo detection system. An ultrasonic pulse is transmitted from the transducer and the two wavelets diffracted form the two end points of the straight-line object are received by three spatially separated receivers. Three stages of signal processing are carried out. At the first stage, the Mother Wavelet operator generates three sets of two-dimensional wavelet coefficients. At the second stage, cross wavelet transforms are performed on wavelet coefficients obtained in the first stage. At the third stage, cross wavelet transforms are performed on cross wavelet coefficients obtained in the second stage. As a result of this three-stage operation, a high-resolution image about the environment is generated and the range and bearing of the two end points of the straight-line object are obtained. A simulation program is developed to investigate the processing algorithm in an ultrasonic detection environment.
\end{abstract}

\section{Introduction}

Ultrasonic non-destructive testing has been widely used in the detection of defects and flaws in materials for several decades ${ }^{1-3}$. Ultrasound is employed in detection because it suffers from relatively low attenuation in solid and liquid when compared to other sources of energy such as electromagnetic waves. Ultrasonic detection in its simplest form is a pulse-echo detection system, where ultrasonic pulses are transmitted and the reflected pulses are detected to determine the location of objects. With more sophisticated signal processing algorithms, the geometrical properties such as size, shape, and orientation, of objects can also be investigated by ultrasonic imaging methods. An inherent problem with ultrasonic detection is the conflict between signal-to-noise ratio and resolution. If good resolution is to be obtained, the transmitted pulse cannot be long in duration. If short ultrasonic pulses are used, their energy is small and hence will result in small signal-to-noise ratio. Pulse compression techniques have been used to overcome this problem. In this paper, the Wavelet Transform and Cross Wavelet Transform are employed to process the ultrasonic signals to obtain a high-resolution image map and then to determine the size and orientation of a straight-line object.

\section{Theory}

In a pulse-echo ultrasonic detection system, a transmitter sends out ultrasonic pulses. These ultrasonic pulses will get reflected when they impinge onto a boundary with acoustic discontinuities $^{3}$. A receiver will pick up the reflected signals and then transform them into other domains such as frequency domain, correlation domain, transfer function domain, and Wigner domain. When the signals are investigated in some other domain, some important features may be emphasized and can be detected by a feature extractor.

The term wavelet refers to an oscillatory wave with a fast decaying amplitude in both the positive- and negative-time directions ${ }^{4}$. A mother wavelet $g(t)$ can serve as a basis function to construct a set of wavelets, which may be employed to approximate a signal. Each element in the wavelet set is obtained by time-scaling the mother wavelet by a parameter $a$ and 
time-shifting the mother wavelet by a parameter $b$. Hence each element can be represented as $\frac{1}{\sqrt{a}} g\left(\frac{t-b}{a}\right)$, where $a>0$ and $b$ can be any value. When $a>1$, the mother wavelet is dilated in time scale; when $a<1$, it is compressed. The factor $\frac{1}{\sqrt{a}}$ keeps the energy of each wavelet element the same irrespective of the values of the parameters $a$ and $b$. If both the time-shifting and time-compressing operations are performed simultaneously, the operation is known as an affine operation. The set of the wavelet elements generated from a mother wavelet is known as a wavelet set. A function $f(t)$ can be represented as a superposition of the wavelet elements in a wavelet set.

Given a mother wavelet $g(t)$ as the kernel, the wavelet transform of a function $f(t)$ can be written as:

$$
W_{g} f(a, b)=\frac{1}{\sqrt{|a|}} \int_{-\infty}^{\infty} f(t) g^{*}\left(\frac{t-b}{a}\right) d t
$$

Where $g^{*}(t)$ represents the complex conjugate of $g(t)$.

The wavelet transform maps a signal $f(t)$ in the time domain to the two-dimensional domain defined by $(a, b)$. The expression $W_{g} f(a, b)$ is known as the wavelet coefficient. A large value of $W_{g} f(a, b)$ implies that the signal $f(t)$ closely resembles the mother wavelet which is time-scaled by $a$ and time-shifted by $b$. The Fourier transform is a special case of wavelet transform with $g(t)=e^{i t}, a=1 / \omega$, and $b=0$. The wavelet transform of the signal $x(t)$ with the signal $y(t)$ as the kernel can be obtained in terms of the individual wavelet transforms of $x(t)$ and $y(t)$ as?

$W_{y} x(a, b)=\frac{1}{c_{g}} \int_{-\infty}^{\infty} \int_{\infty}^{\infty} W_{g} x(s, \tau) W^{*} y\left(\frac{s}{a}, \frac{\tau-b}{a}\right) \frac{d s d \tau}{s^{2}}$

According to Heisenberg's uncertainty principle, the time resolution and frequency resolution cannot be achieved simultaneously. One can only be obtained at the expense of the other. When a signal is analyzed by the short time Fourier transform (STFT), the time window with a duration of $T$ seconds will fix the frequency resolution $\Delta f$ across the entire band at an order of $1 / T \mathrm{~Hz}$. For the analysis of most signals, the relative frequency resolution $\Delta f / f$ is more important than the absolute frequency resolution $\Delta f$. For instance, a frequency resolution of $1 \mathrm{~Hz}$ will be very good at $1 \mathrm{kHz}$, but will be poor at $10 \mathrm{~Hz}$. The time compression factor $a$ in the Wavelet Transform controls the frequency resolution. It is this feature that gives the Wavelet Transform its multi-resolution analysis capability ${ }^{5,6}$. The time shift factor $b$ localizes the features of a signal in the time domain. Consider the case to detect two short bursts of sinusoids that only differ a little from each other in both the time and frequency domains. Conventional STFT cannot give a satisfactory result since time and frequency resolution cannot be achieved simultaneously. With Wavelet Transform, a small value of $a$ can provide good time resolution. At high frequency contents, the frequency resolution will be good enough and hence the two pulses can be separated. Such an approach is most suitable for the analysis of short pulses with high frequency contents. Signals employed in a typical ultrasonic pulse-echo detection system should fall into this category and hence Wavelet Transform would be a suitable processing tool' ${ }^{7}$.

\section{Signal Processing System Design}

In this paper, wideband ultrasonic pulses are transmitted from a point source with equal directivity in all directions to detect the end points of a straight-line object. Three spatially separated receivers are used to capture the reflected signals, as shown in Figure 1.

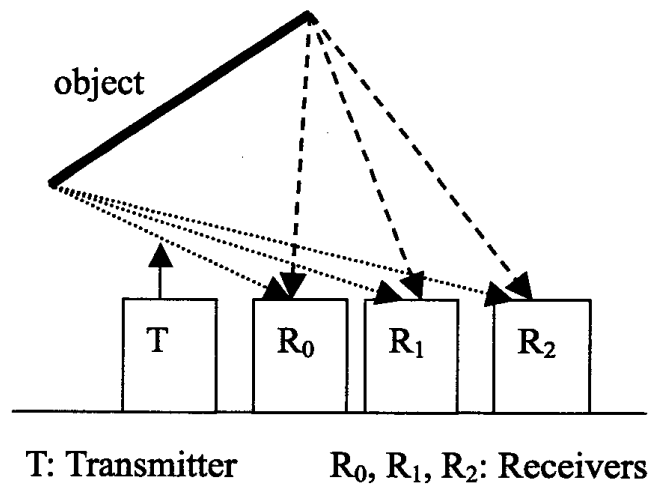

Figure 1 General Set-up

Each receiver receives a signal that is a superposition of the waveforms diffracted by the end points of the object. The signal received by 
the $i^{\text {th }}$ receiver is denoted as $r_{i}(t), i=0,1,2$. These signals are processed in a 3-stage operation. In the first stage, the Wavelet Transforms of the three received signals are calculated as $W_{g} r_{0}(a, b), W_{g} r_{1}(a, b)$ and $W_{g} r_{2}(a, b)$. In the second stage, the two wavelet transforms $W_{g} r_{1}(a, b)$ and $W_{g} r_{0}(a, b)$ are combined to obtain the cross wavelet transform $W_{r_{1}} r_{0}(a, b)$. Similarly, $W_{g} r_{1}(a, b)$ and $W_{g} r_{2}(a, b)$ are combined to obtain $W_{r_{1}} r_{2}(a, b)$. In the third stage, the two cross wavelet transforms obtained in stage 2 are combined to obtain an estimate of the cross wavelet trans $W_{r_{2}} r_{0}(a, b)$. This result represents a high-resolution map of the ultrasonic imaging environment. The 3-stage processing is illustrated in Figure 2.

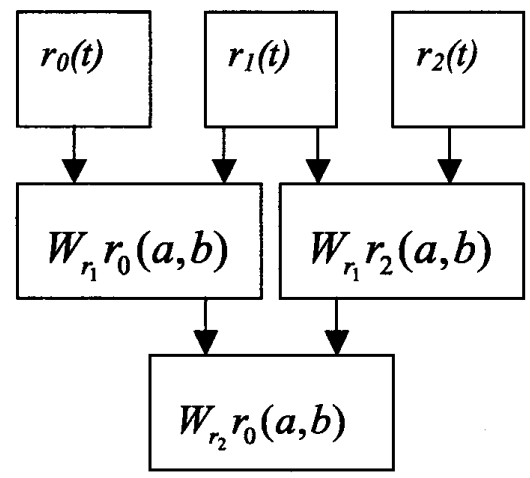

Figure 2 Three-stage processing

\section{Simulation System and Results}

To investigate the effectiveness of the three-stage processing system for ultrasonic detection, a simulation system is set up. In this system, the various parameters of the environment such as positions of the transmitter and the receivers, positions, orientation, and size of the straight-line object to be detected, the mother wavelet waveform, ultrasonic operating frequency, speed of ultrasound, are specified. The wavelet transforms and cross wavelet transforms are then calculated at each of the three stages. Based on the wavelet transform results, the range and bearing of the two end points of the straight-line object are calculated. The simulation results are then compared to the actual values input into the simulation system.

In the first set of simulation data, the straight-line object is 0.1 meter in size lying parallel to the line of the transmitter and the receivers, as illustrated in Figure 3 . The ultrasound velocity is $1000 \mathrm{~m} / \mathrm{sec}$. The operation frequency is $400 \mathrm{kHz}$. The mother wavelet used in the simulation study is the Morlet function.

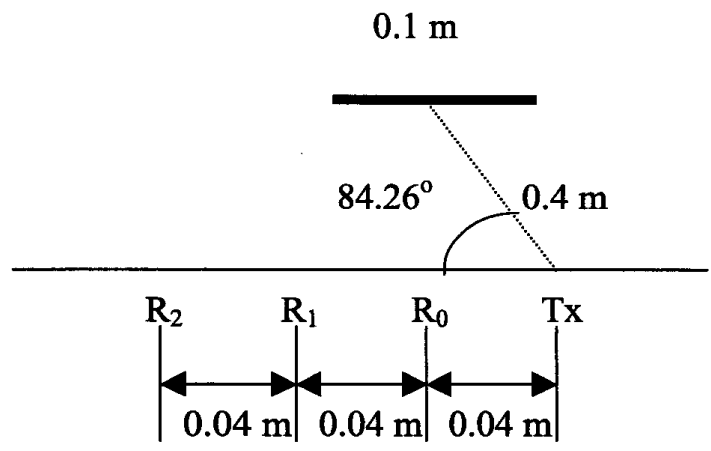

Figure 3 Simulation System Set-up

A cross-section of the cross wavelet transform $W_{r_{2}} r_{0}(a, b)$ obtained at third stage is as shown in Figure 4 below.

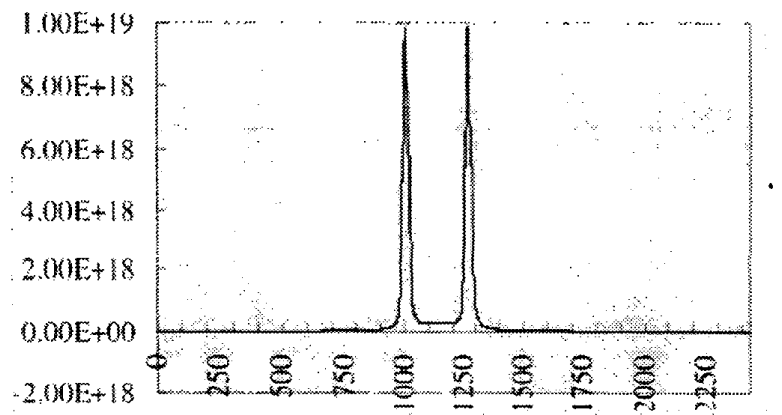

Figure $4-W_{r_{2}} r_{0}(a, b)$ obtained at stage 3

The other results obtained are tabulated below:

\begin{tabular}{|l|l|l|}
\hline & $\begin{array}{l}\text { Actual } \\
\text { Distance }(\mathrm{m})\end{array}$ & $\begin{array}{l}\text { Calculated } \\
\text { Distance }(\mathrm{m})\end{array}$ \\
\hline $\mathrm{R}_{1}$ to $\mathrm{Pt} .1$ & -0.398 & -0.401 \\
\hline $\mathrm{R}_{1}$ to $\mathrm{Pt} .2$ & -0.408 & -0.413 \\
\hline $\mathrm{R}_{2}$ to $\mathrm{Pt} .1$ & -0.399 & -0.402 \\
\hline $\mathrm{R}_{2}$ to $\mathrm{Pt} .2$ & -0.419 & -0.424 \\
\hline
\end{tabular}

\begin{tabular}{|l|l|l|}
\hline & $\begin{array}{l}\text { Actual } \\
\text { Bearing }\left({ }^{\circ}\right)\end{array}$ & $\begin{array}{l}\text { Calculated } \\
\text { Bearing }{ }^{\circ} \text { ) }\end{array}$ \\
\hline $\mathrm{R}_{1}$ to Pt. 1 & 88.56 & 86.8 \\
\hline $\mathrm{R}_{1}$ to $\mathrm{Pt} .2$ & 77.26 & 77.0 \\
\hline $\mathrm{R}_{2}$ to $\mathrm{Pt} .1$ & 85.68 & 84.5 \\
\hline $\mathrm{R}_{2}$ to $\mathrm{Pt} .2$ & 71.9 & 70.7 \\
\hline
\end{tabular}


From these data, the dimension of the straight-line object is calculated as $\mathbf{0 . 1 0 2}$ meter. With an actual size of 0.1 meter, the discrepancy is about $2 \%$.

In a second set of simulation data, the straight-line object is placed $10 \mathrm{~m}$ from the transmitter at a bearing angle of $135^{\circ}$. The object size is $2 \mathrm{~m}$. The ultrasound velocity is $1000 \mathrm{~m} / \mathrm{sec}$. The frequency of operation is 200 $\mathrm{kHz}$. A cross-section of the cross wavelet transform $W_{r_{2}} r_{0}(a, b)$ obtained at third stage is as shown in Figure 5 below.

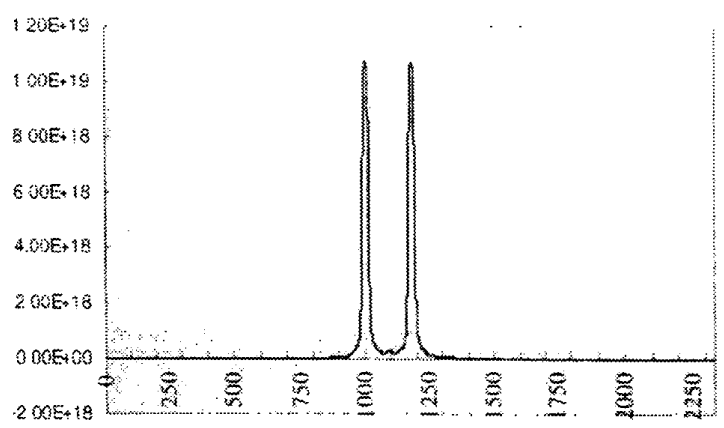

Figure $5-W_{r_{2}} r_{0}(a, b)$ obtained at stage 3

Based on the simulation results, the size of the straight-line object is derived as $1.9 \mathrm{~m}$. Compared to the actual size of $20 \mathrm{~m}$, the discrepancy is about $5 \%$.

\section{Discussion and Conclusion}

A simulation system has been implemented to perform ultrasonic signal detection by a three-stage wavelet transform processor. The two end points of a straight-line object can be located and hence the size and orientation of the object can be determined. The proposed method will be further tested for its performance with respect to different mother wavelets (such as Haar, Shannon, etc.) instead of the Morlet function.

\section{References}

1. Fitting, Dale W., Adler, L.: Ultrasonic Spectral Analysis for Nondestructive Evaluation, Plenum Press, New York, 1981, pp.69-86, 93-110.

2. Johnson, D.H.: 'The Application of Spectral Estimation Methods to Bearing Estimation Problems', Proc. IEEE, 1982, 9(70), pp.1018-1028

3. Chen, H.: Signal Processing and Pattern Recognition in Nondestructive Evaluation of Materials, Springer-Verlag, New York, 1988.

4. Daubechies, I.: 'The wavelet transform, time-frequency localization and signal analysis', IEEE Trans. Info. Theory. 1990, Vol. 36, pp. 961-1005.

5. Laine, Andrew: Wavelet Theory and Application, Kluwer Academic, Boston, 1993.

6. Telolis, Anthony: Computational Signal Processing with Wavelets, Birkhauser, Boston, 1998.

7. Weiss, L.G., and Young, R.K., 'Wideband Spatial Processing with Wavelet Transforms,' Proc. Sixth SP Workshop on Statistical and Array Processing, Oct., 1992, Victoria, B.C., Canada. 